Japan. J. Med. Sci. Biol., 24, 83-91, 1971

\title{
DETERMINATION OF THE HEMOLYTIC ACTIVITY OF VIBRIO CHOLERAE
}

\author{
RIICHI SAKAZAKI*, KAZUMICHI TAMURA* \\ AND MINORU MURASE** \\ * Department of Bacteriology I, National Institute of Health, \\ Shinagawa-ku, Tokyo 141, Japan \\ ** Public Health Research Institute, Ikuta-hu, Kobe 650, Japan
}

(Received: February 18th, 1971)

\begin{abstract}
SUMMARY : There is no definite opinion on the reliability of the hemolysis test for differentiation between classical and El Tor biotypes of Vibrio cholerae, serotype 1, although several improved modifications of the test have been reported. In order to obtain consistent methods for determining hemolytic activity of $V$. cholerae, studies were carried out using 1,222 strains of the vibrio. It was found, in a preliminary study, that peptone and extract produced from animal tissue stimulated hemolytic activity in culture and that heart infusion broth consisting of those two ingredients was one of the suitable basal media for the hemolysis test. No variation among several batches of dehydrated heart infusion broth of Difco products was observed.

Among plain heart infusion broth (HIB), heart infusion broth by Feeley and Pittman (FP-HIB), glycerolized heart infusion broth (HIGB) and glycerolized brain heart infusion broth, the largest number of hemolysispositive tubes were obtained with HIGB. The results given by two agar plates, an aerobic plate of brain heart-thioglycollate-cystine agar (BHTCA), devised by the authors during the course of this study, and an anaerobic plate of heart infusion agar with washed sheep red cells were also compared with those given by HIGB. It was demonstrated that BHTCA was also satisfactory for the hemolysis test of $V$. cholerae.

Thus, when 1,124 strains resistant to polymyxin B and to Mukerjee's phage IV with one exception being sensitive to the latter were tested in HIGB and BHTCA, the hemolytic activity was demonstrated in $99 \%$ of the strains. Classical strains were consistently nonhemolytic in/on these media; no false positive was recognized in any of them.

However, some varying results were recognized between HIGB tube and BHTCA plate. Variation was also given even in the same medium among strains which revealed weakly or no hemolytic activity. From these results, in this paper the joint use of HIGB and BHTCA has been recommended for the determination of hemolytic activity of $V$. cholerae. The test should be repeated if a doubtful or negative result is obtained.

Role of glycerol in HIGB and of thioglycollate and cystine in BHTCA for detection of hemolytic activity was discussed.
\end{abstract}

* 坂崎利一 - 田村和満 (国立予防衛生研究所細菌第一部)

** 村瀬稔 (神戸市立衛生研究所細菌部) 


\section{INTRODUCTION}

Although Vibrio cholerae biotype eltor is differentiated from the classical biotype of the species principally by its production of heat-labile hemolysin, many workers reported inconsistent results of the hemolytic tests (Tanamal, 1959; Mulzerjee, 1963; Finkelstein and Mukerjee, 1963; Roy, Mridha and Mukerjee, 1965). The hemolytic test on cholera vibrio was originated from a study of Greig (1914), who employed classical cholera vibrio and hemolytic non-cholera vibrio but none of hemolytic cholera vibrios. In recent years, however, modifications have been used more frequently than the original Greig test. Feeley and Pittman (1963) recommended to use 24-hr heart infusion broth culture for the test. Barua and Mukherji (1964) described that glycerolized heart infusion broth gave larger number of positive results than the broth without glycerol. Ganguly et al. (1966) reported superiority of glycerolized brain heart infusion broth for the test. On the other hand, deMoore (1963) recommended an anaerobic culture on blood agar plate for detecting hemolytic activity.

The hemolytic activity should be most important to differentiate between classical and El Tor biotypes of $V$. cholerae, since the name $V$. cholerae biotype eltor has been designated for its hemolytic activity (Hugh, 1965; Feeley, 1966). Although a number of other procedures such as tests for chicken red cell agglutination (Finkelstein and Mukerjee, 1963) and for susceptibility to polymyxin B and Mukerjee's phage IV (Mukerjee, 1963) have been prompted for differentiation of El Tor biotype from classical cholera vibrio, the identification should depend upon confirmation of the hemolytic activity, because it does not appear reasonable to include nonhemolytic vibrios in the $\mathrm{El}$ Tor biotype according to the proposal by the International Subcommittee on Taxonomy of Vibrios (Feeley, 1966). In this view point, several procedures of the hemolysis test have been compared to find a reliable one for testing hemolytic activity of $V$. cholerae.

\section{MATERIALS AND METHOdS}

Strains employed: A total of 1,222 strains of $V$. chorelae, all agglutinable by serotype 1 antiserum of the species, were employed in this study. Almost all the strains were isolated from cases of clinical cholera and contacts. Only five strains were originated from water. The geographical areas in which they were collected were: Afganistan, Burma, Guinea, Hongkong, Indonesia, India, Korea, Malaysia, New Guinea, Pakistan, the Philippines, Thailand, and Vietnam. They were isolated in 1945 through 1970. Some of them were obtained from the following scientists: Dr. R. Sen, Calcutta; Dr. S. C. Sanyal, Calcutta; Dr. L. W. Prescott, WHO Scientist; and Dr. M. Nagao, Japan. Of the 1,222 strains, 550 were isolated by the authors in Calcutta in 1969 and 1970.

All strains were plated on Aronson agar to determine the purity and colony morphology. Pure cultures from typical colonies were maintained in Lab-Lemco agar butt at $22 \mathrm{C}$ with four-monthly transfers. All strains were tested for oxidase, glucose fermentation without gas production, lysine decarboxylation, and agglutination with the serotype 1 antiserum of $V$. cholerae, to be confirmed as $V$. cholerae, serotype 1 .

Tube hemolysis test: Heart infusion broth (HIB), heart infusion broth with $1 \%$ 
glycerol (HIGB) and brain heart infusion broth with $1 \%$ glycerol (BHIGB) were used for the test. The basal media were obtained in commercial dehydrated form and the original $\mathrm{pH}(7.4)$ of the broth was unchanged. Two $\mathrm{ml}$ of broth was inoculated with a loopful of an overnight broth culture of each of the strains to be tested. After 24 -hr incubation, $0.1 \mathrm{ml}$ of a $10 \%$ suspension of fresh, washed sheep red cells in saline was added to the broth culture. Hemolysis was also tested by the method of Feeley and Pittman (1963), in which a $0.5 \mathrm{ml}$ portion taken from the bottom of the tube of a $10 \mathrm{ml}$ of a $24-\mathrm{hr}$ culture in heart infusion broth (FP-HIB) in a $16 \mathrm{~mm} \times 150$ $\mathrm{mm}$ tube was mixed with $0.5 \mathrm{ml}$ of $1 \%$ washed sheep red cell suspension. The mixture was incubated in a water bath at $37 \mathrm{C}$ for $2 \mathrm{hr}$ and left in a refrigerator at $4 \mathrm{C}$ overnight. Reading was taken next morning.

Plate hemolysis test: An agar medium, brain heart-thioglycollate-cystine agar (BHTCA), was devised by the authors for the hemolysis test of $V$. cholerae during the course of this study. The BHTCA consisted of $3 \%$ dehydrated medium of brain heart infusion broth, $0.05 \%$ sodium thioglycollate, $0.05 \%$ L-cystine, and $1 \%$ Oxoid agar No. 1. A $10 \%$ solution of sodium thioglycollate in distilled water and a $10 \%$ solution of L-cystine in diluted $\mathrm{HCl}$ at 1:10 were used to prepare the medium. Ingredients added to distilled water were allowed to stand for $15 \mathrm{~min}$ to dissolve the dehydrated medium. The mixture was adjusted to $\mathrm{pH} 7.4$ and autoclaved at $115 \mathrm{C}$ for $15 \mathrm{~min}$ without preboiling. After mixing well and cooling to $50 \mathrm{C}, 5 \mathrm{ml}$ of a $20 \%$ suspension of washed sheep red cells in saline was added to $100 \mathrm{ml}$ of the medium and the mixture was poured into Petri dishes. Special precaution was taken against overheating the basal medium not to decrease the efficiency. The plate test was also performed with heart infusion agar, consisting of commercial dehydrated medium of heart infusion broth and Oxoid agar No. 1, containing $1.5 \%$ of washed sheep red cells (HIA).

Each plate was inoculated with one drop of an overnight heart infusion broth culture with a Pasteur pipet. More than eight cultures were tested on a single plate. The HIA plate was incubated for $24 \mathrm{hr}$ in an anaerobic jar in which the air was replaced with nitrogen gas. The BHTCA plate was incubated at $37 \mathrm{C}$ for $24 \mathrm{hr}$ in an usual manner.

Polymyxin $B$ sensitivity test : The test was carried out using discs containing 50 units of polymyxin B (Eiken) on DST agar plate (Oxoid) on which $0.1 \mathrm{ml}$ of diluted 24 -hr broth culture at 1:100 was streaked. After placing the discs, the plate was kept in an refregerator at $4 \mathrm{C}$ for $2 \mathrm{hr}$ and then incubated at $37 \mathrm{C}$ for 18 to $24 \mathrm{hr}$.

Susceptibility to Mukerjee's phage IV: It was tested at the routine test dilution (RTD) by the method of Mukerjee (1963).

\section{RESULTS}

\section{Media and Cultural Condition for the Hemolysis Test}

Comparison among different products of peptone and extract.

In a liquid medium with $1 \%$ glycerol, different products of peptone and extract were tested for hemolysis with 100 strains of $V$. cholerae resistant to polymyxin B and the phage IV. As shown in Table 1, the ingredients of the medium significantly influenced the detection of the hemolytic activity of the cultures. A larger number of positive tubes were obtained, in general, in broth containing peptone produced 
Table 1. Influence of products of peptone and extract on hemolysis test with 100 strains

\begin{tabular}{lc}
\hline \multicolumn{1}{c}{ Products } & Number of positive \\
\hline Bacto-tryptone & 20 \\
Bacto-casitone & 23 \\
Bacto-peptone & 64 \\
Bacto-proteose peptone & 88 \\
Bacto-proteose peptone No. 3 & 43 \\
Bacto-neopeptone & 73 \\
Bacto-tryptose & 88 \\
Trypticase-BBL & 24 \\
Gelysate-BBL & 21 \\
Biosate-BBL & 21 \\
Polypeptone-BBL & 88 \\
Myosate-BBL & 68 \\
Thiotone-BBL & 89 \\
Beef extract+Thiotone-BBL & 90 \\
Beef extract +Trypticase-BBL & 24 \\
Heart infusion-Eiken+Bacto-peptone & 88 \\
Heart infusion-Eiken+Thiotone-BBL & 100 \\
Heart infusion-Eiken+Bacto-tryptose & 100 \\
Heart infusion-Eiken+Bacto-proteose peptone & 100 \\
Phytone-BBL+Bacto-tryptose & 87 \\
\hline
\end{tabular}

Each ingredient was used in broth $(\mathrm{pH} 7.4)$ containing $1 \%$ glycerol.

from animal tissue, such as Bacto-proteose peptone, Bacto-tryptose and Thiotone-BBL, than in that wit'l peptone from milk casein. When infusion from heart muscle obtained from the Eiken Co. in a dehydrated form was added to the broth, a largest number of positive tubes were recognized, whereas beef extract and soy peptone (Phytone-BBL) did not affect the results. It was confirmed, therefore, that heart infusion broth was the most appropriate medium for the hemolysis test of $V$. cholerae, since the medium contains infusion and peptone produced from animal tissue.

Comparison among products of heart infusion broth.

When the Difco product of heart infusion broth was compared with that from other three manifactures using the broth containing $1 \%$ glycerol, and the 100 strains tested gave positive hemolysis in tubes of Difco broth, while some gave negative results in broth prepared with the latter three. The differences among four batches of the Difco products were much less than those among the manifactures.

Effect of incubation period on detection of the hemolytic activity in HIGB.

Five tubes of HIGB were inoculated simultaneously with each of 50 strains and incubated at $37 \mathrm{C}$. Each tube was tested after incubation for 10, 18, 24, 36, or $48 \mathrm{hr}$. Only a few cultures showed weak hemolysis at 10 and 18 -hr cultures. All were 
positive at $24 \mathrm{hr}$, although the reaction in some tubes was weak. In $36 \mathrm{hr}$ all cultures were positive and the reaction was strong in most tubes. However, the number of positive tubes decreased in $48 \mathrm{hr}$.

Comparison among several batches of BHTCA.

Brain !heart infusion broth was a principle basal medium in BHTCA; heart infusion or nutrient broth could not replace it. Three batches of brain heart infusion broth of Difco and one batch each of the medium from BBL, Eiken and Nissui were used to prepare the basal medium of BHTCA to be compared with the 100 strains. No difference in the reaction was observed among these batches.

\section{Comparison with 1,222 Strains}

In order to determine what difference would result from the use of HIGB and BHTCA, results obtained with these two media were compared with those obtained with HIB, FP-HIB and BHIGB and also with anaerobic plate of HIA containing washed sheep red cells using 1,222 strains of $V$. cholerae. The tests were repeated at least three times and the final results are shown in Table 2.

Table 2. Hemolytic activity of 1,222 strains with different media

\begin{tabular}{|c|c|c|c|c|c|c|c|c|}
\hline \multicolumn{2}{|c|}{ Susceptibility } & \multirow{3}{*}{$\begin{array}{l}\text { Number of } \\
\text { strains }\end{array}$} & \multicolumn{6}{|c|}{ Medium } \\
\hline & & & \multicolumn{3}{|c|}{ Tube } & \multicolumn{3}{|c|}{ Plate } \\
\hline $\begin{array}{l}\text { Polymyxin B } \\
\text { (50u. disc) }\end{array}$ & Phage IV & & HIB & FP-HIB & BHIGB & $\mathrm{HIGB}$ & HIA & BHTCA \\
\hline+ & + & 98 & 0 & 0 & $\begin{array}{c}23 \\
(23)\end{array}$ & 0 & 0 & 0 \\
\hline- & + & 1 & 0 & 0 & 1 & 1 & 1 & 1 \\
\hline- & - & 1,123 & 361 & 481 & $\begin{array}{l}1,095 \\
(43)\end{array}$ & 1,106 & 1,083 & 1,084 \\
\hline
\end{tabular}

Remarks: HIB-plain heart infusion broth, FP-HIB-heart infusion broth by Feeley and Pittman, BHIGB - b:ain heart infusion broth with $1 \%$ glycerol, HIGB - heart infusion bioth with $1 \%$ glycerol, HIA - heart infusion agar with washed red cells (anaerobic plate), BHTCA - brain heart-thioglycollate-cystine agar.

Figure in parentheses indicates number of false positive tubes.

As shown in the table, 98 of the 1,222 strains were susceptible to polymyxin B and the phage IV, and none of the 98 strains gave positive reaction in any of the media except BHIGB. In BHIGB cultures, 23 of the 98 strains cleared the red cell suspension added to the culture. However, the color of those clear mixtures in the tubes were brown which was quite different from red color of true hemolysis. In addition to this, the hemolytic activity of the culture remained after heating at $60 \mathrm{C}$ for $30 \mathrm{~min}$. It was considered that the reaction given by the 23 cultures was the "hemodigestion".

The remaining 1,124 were resistant to polymyxin B and the phage IV, except for one sensitive to the phage. Of the 1,124 strains, 1,118 were hemolytic in one or more media. From Table 2, variability in the hemolytic activity in the five media 
used can readily be seen.

Of the three media containing heart infusion broth, HIGB gave positive tubes two or three times more than those of plain HIB and FP-HIB. Hemolytic strains in HIB or FP-HIB or both also gave constant positive hemolysis in the HIGB tube. There was no strain giving a positive reaction in HIB and FP-HIB and negative in HIGB. BHIGB also gave a number of positive tubes. In this broth, however, 43 of 1,095 positive tubes showed the activity after heat treating at $60 \mathrm{C}$ for $30 \mathrm{~min}$, although hemolytic activity was inactivated by treating positive tubes of the other broth cultures.

By the plate method, no different hemolytic activity between BHTCA and anaerobic HIA with red cells was observed. In addition, the hemodigestion of ten recognizable on the aerobic blood agar cultures was not observed on these two agar plates.

When the tests were repeated, the same results were constantly obtained with HIB. However, more or less variation in the results by individual strains was observed with the other media. In general, variation occurred in cultures giving weakly hemolytic reaction in those media, although consistently positive reactions were given by strongly hemolytic strains and consistently negative reaction by all strains susceptible to polymyxin $\mathrm{B}$ and the phage IV. There was little variation among the results with each individual strain in FP-HIB and BHTCA, but more variation in HIGB.

The strains giving negative or weakiy positive results in either or both of HIGB and BHTCA and resistant to polymyxin B and the phage IV were tested repeatedly at 2 or 3 days' intervals in each medium. They were initially plated onto Aronson agar. Three or more with morphologically different colonies were examined. As shown in Table 3, cultures from one or more colonies of 41 of 47 strains which were nonhemolytic in the initial HIGB tube showed hemolytic activity in HIGB and so did those of 18 of 58 nonhemolytic strains on the initial BHTCA plate on BH.TCA. Cultures from six of the 1,124 strains resistant to polymyxin $\mathrm{B}$ and the phage IV except one sensitive to the latter were constantly nonhemolytic in both media although the colonial selection and the hemolysis test were repeated more than five times.

Table 3. Results of retest with strains initially giving negative reaction in the same medium

\begin{tabular}{clcr}
\hline \multirow{2}{*}{ Number of strains } & \multicolumn{3}{c}{ Hemolysis test } \\
\cline { 2 - 4 } & Medium & + & - \\
\hline \multirow{2}{*}{47} & HIGB tube & 41 & 6 \\
58 & BHTCA plate & 18 & 40 \\
\hline
\end{tabular}

A total of 1,072 hemolytic strains in HIGB gave also positive reactions on BHTCA, but the degrees of hemolysis in both media by an individual strain were not always the same. On the other hand, 34 strains were hemolytic only in HIGB tube, whereas 12 only on BHTCA plate. According to their hemolytic attitudes in both media, the 1,124 strains were divided into four categories as indicated in Table 4 . 
Table 4. Grouping 1,124 strains by their hemolytic activities in HIGB tube and on BHTCA plate

\begin{tabular}{ccc}
\hline \multirow{2}{*}{ Number of strains } & \multicolumn{2}{c}{ Hemolytic activity } \\
\cline { 2 - 3 } & HIGB tube & BHTCA plate \\
\hline 1,072 & + & + \\
34 & + & - \\
12 & - & + \\
6 & - & - \\
\hline
\end{tabular}

Of the 1,222 strains studied here, 550 were isolated by the present authors during the cholera studies in Calcutta (1969 and 1970) under the joint auspices of the Council of Medical Research, the West Bengal Government Health Department and the World Health Organization. The 550 strains were tested for hemolytic activity at that time of isolation by using HIA plate containing washed sheep red cells, under an anaerobic condition and also in HIGB tube. All the strains, except 12 which were sensitive to polymyxin $B$ and the phage IV, lysed red cells on HIA plate, and 449 and 83 of these strains gave weak and strong reaction, respectively, in HIGB tube. When these strains were retested for the hemolytic activity after 5 to 7 months, the same results as those at the initial time were given on the anaerobic HIA plate. On the other hand, these strains showed some variation in their hemolytic activity in HIGB tubes when kept in stock for 5 to 7 months. Of the 532 strains which gave weakly positive reaction in the initial HIGB tubes, 160 exhibited strong hemolysis in the tube, 13 of 18 strains which gave negative result in the initial tubes showed weakly positive results in the tube, and no variation of a degree of the reaction was observed with the remaining strains.

\section{DISCUSSION}

This study indicates that HIGB is supperior to HIB and BHIGB for the tube hemolysis test with $V$. cholerae and that incubation for more than $24 \mathrm{hr}$ in HIGB is required to achieve satisfactory results. The plate method of the test using BHTCA was also appropriate because it gives a larger number of true positive and no false positive results and it does not require anaerobic cultivation of the plate.

It seems that glycerol in HIGB and glucose in BHTCA are important components for hemolysis by cholera vibrio. Sen and Shrivastava (1970) described that " the hemolytic property can be induced in chicken-erythrocyte-agglutinating strains by adding glycerine to the growth medium........ But, this would appear to be an artificial method of manifesting a property not originally apparent". The present authors, however, could not accept their opinion. It should be noted that the hemolytic activity could not be induced in any strain sensitive to polymyxin B and the phage IV even in HIGB. The hemolytic activity observed in HIGB and BHTCA was a property of only such strains that were resistant to polmyxin B and the phage IV. Feeley and Pittman (1963) demonstrated the maximum hemolytic activity at $\mathrm{pH}$ j.5 to 7.0 and the optimum $\mathrm{pH}$ of media for hemolysin production at $\mathrm{pH} 7.1$ to 7.4. 
It seems, therefore, that $1 \%$ glycerol in HIGB and $0.2 \%$ glucose in BHTCA act as a stabilizer of $\mathrm{pH}$. Acid produced from those carbon compounds possibly neutralizes an alkaline $\mathrm{pH}$ of the medium brought about by growth of vibrios. On the other hand, it is known that false positive reaction may be obtained when the medium contains a sufficient amount of carbohydrate to lower the $\mathrm{pH}$ to 5.5 (Vassiladis, 1935). It was recognized, however, that positive reaction in HIGB and BHTGA was not due to acid hemolysis, since the hemolytic activity of the HIGB culture was not observed in heated culture and the $\mathrm{pH}$ values of the cultures were 7.0 to 7.2 after 24-hr incubation.

Feeley and Pittman (1963) suggested that the rapid disappearance of hemolysin from the recently isolated South East Asian cultures may have been due to the rapid autodestruction. Wake and Yamamoto (1966) reported a hemolysin destructive factor produced concomitantly with hemolysin by $\mathrm{El}$ Tor cholera vibrios. In a preliminary study on BHTCA, the authors found that some enzymatic activity of the vibrios such as gelatin hydrolysis decreased when they were cultured under an anaerobic condition or in a medium containing some carbohydrate. It is possible that such condition in HIGB and BHTCA inhibits the activity or production of the hemolysin destructive factor.

Sen and Shrivastava (1970) emphasized that the hemolytic activity of $V$. cholerae is a constant property. It may be partially true. It should be mentioned, however, that results of the tests are not necessarily consistent. In this study, the majority of strains gave consistently strong positive reactions, but some strains gave different reactions when the tests were repeated. In addition to this, Barua and Gomez (1967) reported that different colonies from a given culture varied in their hemolytic activity. Their finding has also been confirmed in this study.

Sen and Shrivastava (1970) cautioned that the blood agar plate method may show false positive as hemodigestion may mistakenly be recorded as hemolysis. It should be emphasized, however, that no hemodigestion was observed on agar plate containing washed sheep red cells so for as the plate was incubated at $37 \mathrm{C}$ for 24 hr under anerobic condition, non on BHTCA plate under aerobic condition. Furthermore, no strain resembling the classical biotype of $V$. cholerae showed hemolytic activity on these plate. The authors believe, therefore, that the plate method is also appropriate for the hemolysis test of $V$. cholerae, although Sen and Shrivastava emphasized the tube method for the hemolysis test.

It should be noted that with some strains the results in HIGB tube were different from these on BHTCA plate. This finding suggests that the hemolysin produced by El Tor cholera vibrios may consists of at least two elements, but the search for such elements has not been carried out.

From these findings it is recommended that the tube test in HIGB is used jointly with the plate test on BHTCA for detection of hemolytic activity of $V$. cholerae. When BHTCA is not available, HIA with washed sheep red cells can also be used under an anaerobic condition. If a negative result is obtained with either the tube or plate method, the test should be repeated.

As mentioned above, Barua and Gomez (1967) described the dissociation of nonhemolytic colonies from hemolytic cultures. Such colonical dissociation was also observed in this study. Several nonhemolytic, polymyxin B- and phage IV-resistant strains found in the authors' collection may be such nonhemolytic mutants derived 
from hemolytic strains. At present, however, such nonhemolytic strains should not be designated as $V$. cholerae biotype eltor. The name "biotype eltor" was proposed to designate hemolytic strains of $V$. cholerae by the International Subcommittee on Taxonomy of Vibrios. The biotype for such nonhemolytic strains which do not belong to the classical biotype should be determined with careful consideration.

\section{ACKNOWLEDGEMENTS}

The authors are grateful to Drs. R. Sen, S. C. Sanyal, and M. Nagao for providing the strains employed. Their grateful acknowledgment is also made to the World Health Organization for the grant to one of us (R. S.) for a part of this study.

\section{REFERENCES}

BARUA, D. AND GomeZ, C. Z. (1967): Observation on some test commonly employed for the characterization of El Tor vibrios. Bull. WHO, 37, 800-803.

BARUA, D. AND MukherJi, A. C. (1964): Observation on the El Tor vibrios isolated from cases of cholera in Calcutta. Bull. Cal. Sch. Trop. Med., 12, 147-148.

FeELEY, J. C. (1966): Minutes of IAMS Subcommittee on Taxonomy of Vibrios. Intern. J. Syst. Bacteriol., 16, 135-142.

Feeley, J. C. And Pittman, M. (1963): Studies on the haemolytic activity of El Tor vibrios. Bull. WHO, 28, 347-356.

Finkelstein, R. A. AND MukeRJee, S. (1963): Haemagglutination: A rapid method for differentiating $V$. cholerae and El Tor vibrios. Proc. Soc. Exptl. Biol. Med., 112. 355359.

Ganguly, R., Ghosh, A. K., De, S. P. AND Shrivastava D. L. (1966): Haemolysis in vibrios. Ind. J. Med. Res., 54, 15-23.

GrEIG, E. D. W. (1914): The haemolytic action of Indian strains of cholera and choleralike vibrios. Ind. J. Med. Res., 2, 623-647.

HUGH, R. (1965): A comparison of Vibrio cholerae Pacini and Vibrio eltor Pribram. Intern. Bull. Bact. Nomen. Tax., 15, 61-68.

MOORE, C. E. de (1963): A non-hemolytic El Tor vibrio as the cause of an outbreak of paracholera in West New Guinea. Trop. Geogr. Med., 15, 97-105.

MUKERJEE, S. (1963): The bacteriophage susceptibility test in differentiating Vibrio cholerae and Vibrio El Tor. Bull. WHO, 28, 333-336.

Roy, C., Mridha, K. AND MukerJeE, S. (1965): Action of polymyxin on cholera vibrios. Techniques of determination of polymyxin-sensitivity. Proc. Soc. Exptl. Biol. Med., $119,893-896$.

Sen, R. AND Shrivastava, D. L. (1970): A note on designating strains as Vibrio cholerae biotype eltor. Ind. J. Med. Res., 58, 174-179.

TANAmal, S. T. W. (1959): Notes on paracholera in Sulawesi (Celebes). Am. J. Trop. Med. Hyg., 8, 72-78.

VASSILADIS, P. C. (1935): Compt. Rend. Soc. Biol. (Paris), 119, 332 (quoted by Feeley and Pittman, 1963).

WAKE, A. AND YAmamoto, M. (1966): Haemolysin destructive factor of $V$. cholerae ( $V$. comma). J. Bacteriol., 91, 461. 